\title{
The 4m International Liquid Mirror Telescope (ILMT)
}

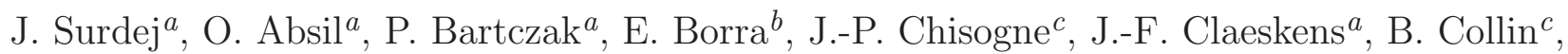 \\ M. De Becker ${ }^{a}$, D. Defrère ${ }^{a}$, S. Denis ${ }^{c}$, C. Flebus ${ }^{c}$, O. Garcet ${ }^{a}$, P. Gloesener ${ }^{c}$, C. Jean ${ }^{a}$, P.

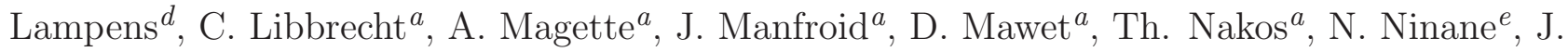 \\ Poels $^{a}$, A. Pospieszalska ${ }^{a}$, P. Riaud ${ }^{a}$, P.-G. Sprimont ${ }^{a}$, J.-P. Swings ${ }^{a}$ \\ ${ }^{a}$ Institut d'Astrophysique et de Géophysique, Liège University (IAGL), Belgium \\ ${ }^{b}$ Laval University, Canada \\ ${ }^{c}$ Advanced Mechanical and Optical Systems (AMOS), Liège, Belgium \\ $d$ Royal Observatory of Belgium \\ ${ }^{e}$ Centre Spatial de Liège (CSL), Liège University, Belgium
}

\begin{abstract}
The entire funding has recently been obtained in Belgium for the construction of a $4 \mathrm{~m}$ Liquid Mirror Telescope. Its prime focus will be equipped with a semi-conventional glass corrector allowing to correct for the TDI effect and a thinned, high quantum efficiency, $4 \mathrm{~K} \times 4 \mathrm{~K}$ pixel equivalent CCD camera. It will be capable of subarcsecond imaging in the i'(760 nm) and possibly r', g' band(s) over a field of $\sim 30$ ' in diameter. This facility will be entirely dedicated to a deep photometric and astrometric variability survey over a period of $\sim 5$ years. In this paper, the working principle of liquid mirror telescopes is first recalled, along with the advantages and disadvantages of the latter over classical telescopes. Several science cases are described. For a good access to one of the galactic poles, the best image quality sites for the ILMT are located either in Northern Chile (latitude near $-29^{\circ} 30^{\prime}$ ) or in North-East India (Nainital Hills, latitude near $+29^{\circ} 30^{\prime}$ ). At those geographic latitudes, a deep (i' $=22.5$ mag.) survey will approximately cover 90 square degrees at high galactic latitude, which is very useful for gravitational lensing studies as well as for the identification of various classes of interesting galactic and extragalactic objects (cf. microlensed stars, supernovae, clusters, etc.). A description of the telescope, its instrumentation and the handling of the data is also presented.
\end{abstract}

Keywords: liquid mirror telescope, CCD camera, gravitational lensing

\section{INTRODUCTION}

As we know, a perfect paraboloid represents the ideal reference surface for an optical device to focus parallel light rays into a single point. It is therefore of the highest importance that Cappoci already realized in 1850 that the surface of a liquid in a cylindric container, in rotation around its vertical axis, takes the shape of a paraboloid under the constant pull of gravity and the centrifugal acceleration (Mailly 1872). Indeed, the paraboloid surface arises because a liquid surface always sets itself perpendicularly to the net acceleration it experiences locally, which in this case becomes increasingly steeper with distance from the central axis. As an example, for an angular velocity of 33 rotations per minute, corresponding to that of an old gramophone, a focal length of approximately $40 \mathrm{~cm}$ is obtained. Considering a typical turntable with a diameter of $20 \mathrm{~cm}$, the resulting liquid mirror is characterized by a focal ratio $\mathrm{f} / \mathrm{D} \sim 2$. For large mirrors of practical interest, the periods of rotation are of the order of $5-10$ seconds and the linear velocities at the rims of the mirrors range between 5 and $10 \mathrm{~km} / \mathrm{h}$.

The surface of a spinning liquid can therefore be used as the primary mirror of a telescope. Following the suggestion that modern technology (Borra 1982) gives us tracking techniques that render liquid mirrors useful to astronomy, research and development programs were begun to assess the feasibility of the concept. Mirrors

Further author information: send correspondence to Jean Surdej, IAGL, Allée du 6 Août 17, B-4000 Liège, Belgium E-mail: Surdej@astro.ulg.ac.be 
up to a diameter of $2.5 \mathrm{~m}$ were extensively tested and showed the high surface quality of such mirrors (Borra et al. 1992; Borra, Content \& Girard 1993; Ninane \& Jamar 1996; Girard \& Borra 1997). For a well tuned mirror, the root mean square values of the deviations from a perfect paraboloid are typically $\lambda / 20$. It must be noted that liquid mirrors have been tested by several independent labs (Centre Spatial de Liège, University of British Columbia, Laval University).

Liquid mirror telescopes (hereafter LMTs) cannot be tilted and hence cannot track the way conventional telescopes do. To "track" with imagery, one relies on a technique called "time delayed integration" (TDI) also known as drift scan, that uses a CCD detector that tracks by electronically stepping its pixels. The information is stored on disk and the night observations can be coadded with a computer, resulting into longer integration time images : this has been demonstrated (Hickson et al. 1994) with a $2.7 \mathrm{~m}$ diameter liquid mirror telescope. Conversely, the difference image between a reference one characterized by a high $\mathrm{S} / \mathrm{N}$ and images obtained every night may easily lead to the identification of photometrically and/or astrometrically variable objects, including variable point-like components superimposed on extended objects (cf. multiply imaged quasars, supernovae, ...).

Why are we interested in liquid mirror telescopes, considering their limitations? The main reason comes from the fact that based upon existing technology, fairly large $(\mathrm{D} \leq 6 \mathrm{~m})$ liquid mirrors (LMs) can be constructed at very low costs. The low cost ( 2 orders of magnitude less than an equivalent classical telescope) makes it possible for a small team of astronomers to have their own large telescope working full-time on a specific project. This is in practice not conceivable with expensive classical telescopes. Some research projects (e.g. time consuming surveys, long term photometric monitoring programs) simply cannot be envisioned with classical telescopes but become possible with LMTs. This is particularly true for the types of research where the region of the observed sky is not very important (e.g. cosmology). Furthermore, the quality of the recorded observations is optimal at zenith since both the seeing and transparency are the best there, at all times. Moreover, the observing efficiency is very high; indeed, when observing each night the same strip of sky, there is no time lost for slewing, field acquisition, readout times, etc. And of course, no Observing Programme Committee may hamper the continuous flow of data taking! Let us finally note that flat fielding and defringing are much more accurate than during classical observations since the images are actually formed by averaging the signal over entire CCD columns (in the direction of the scan). Among the apparent disadvantages, let us recall that one can only observe at zenith a strip of constant declination. At a latitude of $\pm 29^{\circ} 30$, a band of half a degree covers 156 square degrees, with 88 square degrees being covered at high galactic latitude $\left(|b I I|>30^{\circ}\right)$. The nightly integration times are rather short, typically $\sim 90 \mathrm{sec}$. but as already stated before it is always possible to coadd data from selected nights in order to get longer integration times. The volume of data collected every night is also quite impressive : typically $\sim 20$ Gbytes of data per night in our case.

In order to qualify, or quantify the scientific output of the technique, one should mention that over 65 nights of observations have been obtained by Hickson \& Mulrooney (1997; see also the paper by Cabanac, Borra \& Beauchemin (1998) analyzing the observations obtained with the NASA 3m liquid mirror telescope), demonstrating that LMTs are sufficiently robust for high quality astronomical observations.

Our project has clear scientific and technical goals. We intend to obtain astronomical data and carry out scientific projects as described in the following section. Technically, we wish to gain experience with the running of an LMT during an extended period of time ( $\sim 5$ years $)$ in a remote site. It represents one step in the development and demonstration of the LM technology in astronomy. We expect to pursue with larger LMTs and possibly multiple LMTs.

\section{SCIENCE WITH THE ILMT}

Given the unique capability of Liquid Mirror Telescopes which can be entirely dedicated to specific observational projects, also considering their very low cost $(\sim 2$ million Euros $)$, it was felt important to gather members from the astronomical community at large to discuss all potential astrophysical and cosmological applications of such telescopes. To fulfill this goal, a workshop entitled "Science with Liquid Mirror Telescopes" was organized at the 
Observatory of Marseille on April 14-15, 1997. Approximately 25 scientists from all over the world participated to this meeting. At the end of the workshop, a general consensus emerged about the need for constructing and operating a 4m International Liquid Mirror Telescope (ILMT) in a good astronomical site in order to achieve several dedicated projects of high scientific interest. A detailed account of the main science drivers that justify building an ILMT as an astronomical research instrument may be found in the proceedings of that meeting (see the URL http://wood.phy.ulaval.ca/workshop/Wproceed.html).

The advantages and limitations of this unusual type of telescope constrain the science that is feasible and thus the science requirements. A short list of the science drivers namely includes:

- statistical determination of the cosmological parameters $H_{0}, q_{0}$ and $\lambda_{0}$ based upon surveys for multiply imaged quasars;

- statistical determination of these same cosmological parameters based upon surveys for supernovae;

- search for quasars and observational studies of large scale structures;

- trigonometric parallaxes of faint nearby objects (e.g. brown dwarfs, etc.);

- detection of high stellar proper motions to probe a new range of small scale kinematics (stars, transneptunian objects, etc.);

- astrometry of multiple star systems;

- a wide range of photometric variability studies (cf. photometry of stars, RR Lyrae, micro-lensing effects, photometry of variable AGN over day to year time scales, etc.);

- detection of low surface brightness and star-forming galaxies, and other faint extended objects (galactic nebulae, supernovae remnants, etc.);

- galaxy clustering and evolution;

- serendipitous phenomena;

- finally, production of a unique database for follow up studies with $8 \mathrm{~m}$ class telescopes (cf. VLT, Gemini, Keck, ...).

Because of space limitation for this paper, we propose to only describe one specific project.

\section{Gravitational lens studies with an LMT}

Given the still very small number $(<100)$ of presently known multiply imaged quasars, almost randomly distributed over the sky, the probability to observe even only one of these within the $\sim 30$ ' zenithal field of view of an LMT is virtually zero. Therefore, the observational strategy for studies of gravitational lensing effects with an LMT rather consists in first surveying a sky area as deep (i' 24) and as wide as possible. Gravitational lens candidates ought to be identified based upon their complex morphologies (several variable point-like components superimposed over an extended object, i.e. the lensing galaxy). In our project, the extent of the field of view is restricted by the size (cf. $4000 \times 4000$ pixels) of the thin $\operatorname{CCD}(\mathrm{s})$ placed at the LMT prime focus. For several obvious reasons (access to the south or north galactic pole, good image quality, ...), best site locations for such an LMT are somewhere in the Atacama desert or in North-East India (Nainital Hills). At a latitude near $\pm 29.5^{\circ}$, a deep LMT survey would cover $\sim 90$ square degrees at high galactic latitude, specially useful for the gravitational lensing (GL) studies proposed here, for the identification of various classes of interesting extragalactic objects (cf. galaxies, clusters, supernovae, etc. at high redshift) and subsequent follow-up observations with $8 \mathrm{~m}$ class telescopes (VLT, Gemini, Keck, ...). At a latitude near $-29.5^{\circ}$, such a survey would in addition provide unique data for studies of the galactic center, galactic structure and stellar populations, including the detection of micro-lensed galactic objects, accurate measurements of stellar proper motions and possibly trigonometric parallaxes useful for the detection of faint red, white, brown dwarfs, halo 
stars and other very low mass stars.

We very briefly recall hereafter several of the well known astrophysical and cosmological applications based upon gravitational lensing effects. When a foreground galaxy (the macro-lens) produces multiple images of a background quasar, it is expected that time delays will become measurable between the light travel times of photometric variations of the quasar along the different trajectories. Such measurements offer a unique opportunity of deriving the value of the Hubble parameter $H_{0}$ which is inversely proportional to the observed time delays (Refsdal 1964) and/or constraining the mass distribution of the lens. In addition, those macro-images are most of the time seen through rather dense parts of the galaxy and there is a good chance that one or several macro-images are affected by micro-lensing (Chang and Refsdal 1979). The micro-lens is a star (or several stars) of the galaxy, acting as a magnifying lens with a very small "field of view" (typically of the order of one micro-arcsec), which produces a more or less intricate network of micro-caustics (cf. Kayser 1992 for a review on micro-lensing). When the light beams coming from different regions of the source cross this network, they get differently amplified, according to their sizes and locations. There will thus result a differential amplification of the different components of the quasar. For instance, in the spectrum of a micro-lensed quasar image, the optical continuum will be more amplified than the Broad Line Region (BLR) which has a larger extent. Due to relative proper motions, this phenomenon varies on a time scale of a few months or years and produces characteristic light curves (and very likely variable spectroscopic line profiles for the broad emission-lines). Of course, the shape of these curves depends on the size of the source. A spectroscopic monitoring of such micro-lensed QSO images, first identified on the basis of an LMT imaging survey, with $8 \mathrm{~m}$ class telescopes will thus allow probing the structure and size of the continuum source, as well as the distribution in size (with an angular resolution of the order of $10^{-6}$ arcsec) and velocity of the BLR clouds.

From previously published statistical GL studies (Surdej et al. 1993, see also Claeskens and Surdej 2002, Kochanek et al. 2004), it is easy to derive the observational requirements to identify within an LMT direct imagery survey a large number (e.g. 50) of multiply imaged quasars. Given a flux limited sample of quasars down to the limiting magnitude $B_{\text {lim }}$, the expected number of multiply imaged quasars $N\left(S, B_{l i m}\right)$ over a sky area $S$ (expressed in square degrees) is easily found to be a function of the macro-lensing optical depth for galaxies to produce multiple images, of the magnification bias, of the number counts of quasars $N_{q}\left(B_{\text {lim }}\right)$ and of the surveyed area $S$. Imposing $N\left(S, B_{\text {lim }}\right)=50$ and adopting, for sake of simplicity, a representative value for the quasar redshift $z_{q}=2$, we have illustrated in Figure 1 the resulting sky area $S$ to be surveyed as a function of $B_{\text {lim }}$. Also shown in this figure are the results for the values of the cosmological parameters $\Omega_{0}=0, \lambda_{0}=0$ and $\Omega_{0}=0, \lambda_{0}=1$ and, finally, the results expected for $\Omega_{0}=1, \lambda_{0}=0$, assuming that the number counts of quasars at faint magnitudes do not flatten out, as suggested by Hawkins and Véron (1995). Similarly, we have illustrated in Figure 2 the total number of quasars $N_{q}^{\text {Total }}$ expected in the surveyed sky area $S$ over which 50 new cases of GL systems ought to be identified.

We conclude that, even under the most "unfavourable" cosmological conditions (i.e. $\Omega_{0}=1, \lambda_{0}=0$ ), a realistic sky area $S$ to be surveyed in order to identify 50 new lenses down to limiting magnitudes $B_{\text {lim }}<24$ can be easily probed with a $4 \mathrm{~m}$ LMT (typically $S<60$ square degrees at high galactic latitude). In this case, a total of approximately 20,000 quasars will also be identified. From the observed numbers of detected lenses and quasars in such a deep and complete survey, we should be able to independently infer the most realistic values for the cosmological parameters $\Omega_{0}$ and $\lambda_{0}$, as well as precisely characterize the luminosity function and number counts of quasars as a function of redshift and magnitude, respectively. Note that observational searches for multiply imaged quasars among highly luminous ones have prevented in the past to use a complete QSO reference sample and introduced many other ill-defined biases in the statistical estimates of the various physical (cf. the efficiency for galaxies to produce multiply imaged quasars) and cosmological parameters $\left(\Omega_{0}, \lambda_{0}\right)$.

As the observations will be carried out using a $4 \mathrm{~m}$ LMT in the drift-scan mode (single integrations of 90 sec), this will ensure obtaining excellent flat-fields as well as defringing corrections. Therefore, considering the case of point sources with a seeing of $0.7 \operatorname{arcsec}$, a photometric $S / N \sim 5$ and combining a number of $N$ repeated scans, the expected magnitude limits $M_{l i m}$ will be those listed in table 1 , for various broad band filters. These data are based on our own simulations and from observations achieved with the NASA 3m LMT. The gravitational lens systems will directly be selected from those quasar candidates revealing a peculiar image 


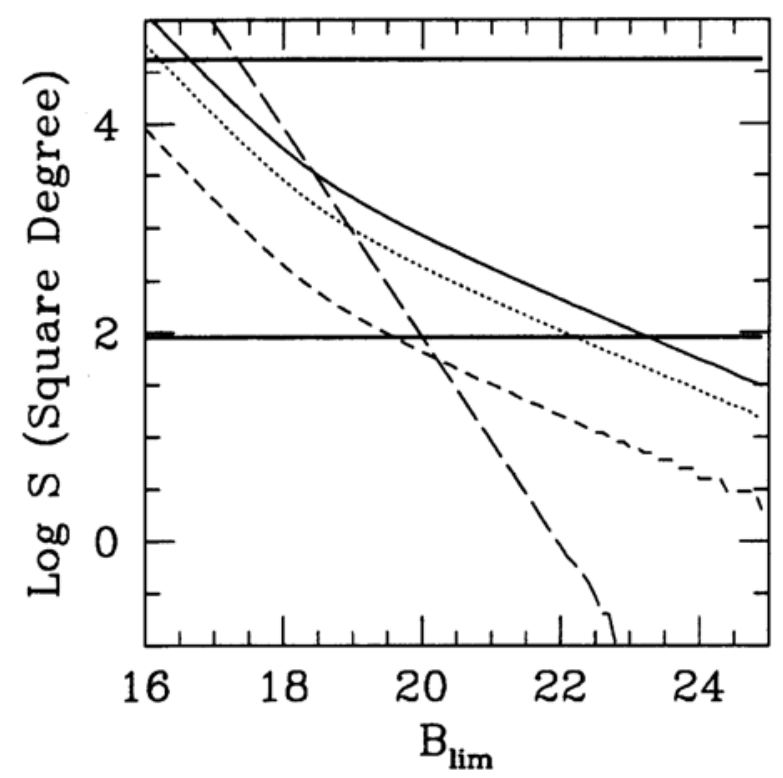

Figure 1. Sky area $S$ to be surveyed as a function of the limiting magnitude $B_{l i m}$ to identify 50 multiply imaged quasars. The two horizontal lines refer to limits set by the whole sky area and a field of 90 square degrees (see text). For simplicity, a representative value of $z_{q}=2$ was adopted for the quasar redshift and, unless stated otherwise, the number counts of quasars are taken from Hartwick and Schade (1990). The different curves refer to $\Omega_{0}=1, \lambda_{0}=0$ (full), $\Omega_{0}=0, \lambda_{0}=0$ (dotted), $\Omega_{0}=0, \lambda_{0}=1$ (dashed) and, finally, $\Omega_{0}=1, \lambda_{0}=0$ (long-dashed) with the number counts of quasars from Hawkins and Véron (1995). Note that for $B>22$ (resp. $B>21$ ), these curves are extrapolations from the Hartwick and Schade (resp. Hawkins and Véron) number counts of QSOs. Conversely, the LMT survey will help in defining more precisely the number counts of quasars at very faint magnitudes, resulting in a better defined and complete sample of QSOs.

\begin{tabular}{|l|l|l|}
\hline Filter & Number of scans & $M_{\text {lim }}$ \\
\hline U & 15 & 24.5 \\
B & 3 & 24.5 \\
V & 6 & 24.5 \\
R & 4 & 23.5 \\
I & 6 & 23.5 \\
Gunn-z & 2 & 22.3 \\
\hline
\end{tabular}

Table 1. Broad band filter, requested number of scans and corresponding limiting magnitude achievable for the detection of point-like sources with a $S / N=5$ photometric accuracy.

morphology (cf. multiple photometrically variable point-like components superimposed on an extended object). Other astrophysical and cosmological applications relying on the studies of the LMT lenses include tracing the luminous and dark matter in the Universe, setting limits on the cosmological density of compact objects with mass $\geq 10^{10} M_{\odot}$ and probing the extinction law of external galaxies responsible for differential reddening between multiple macro-lensed QSO images. In addition, such an extragalactic LMT survey will lead to the discovery of interesting variable stars (cf. distant RR Lyraes to trace the limits on the stellar component of the halo), supernovae, galaxies, clusters at high redshifts and to a large sample of approximately 20,000 quasars down to $B \sim 24$, identified on the basis of their short-, medium- and large- timescale photometric variability. They will provide a unique grid of light probes to study the morphology, structure and size of large scale structures (heavy elements and hydrogen) in the Universe at scales ranging from several Mpcs up to hundreds of Mpcs. 


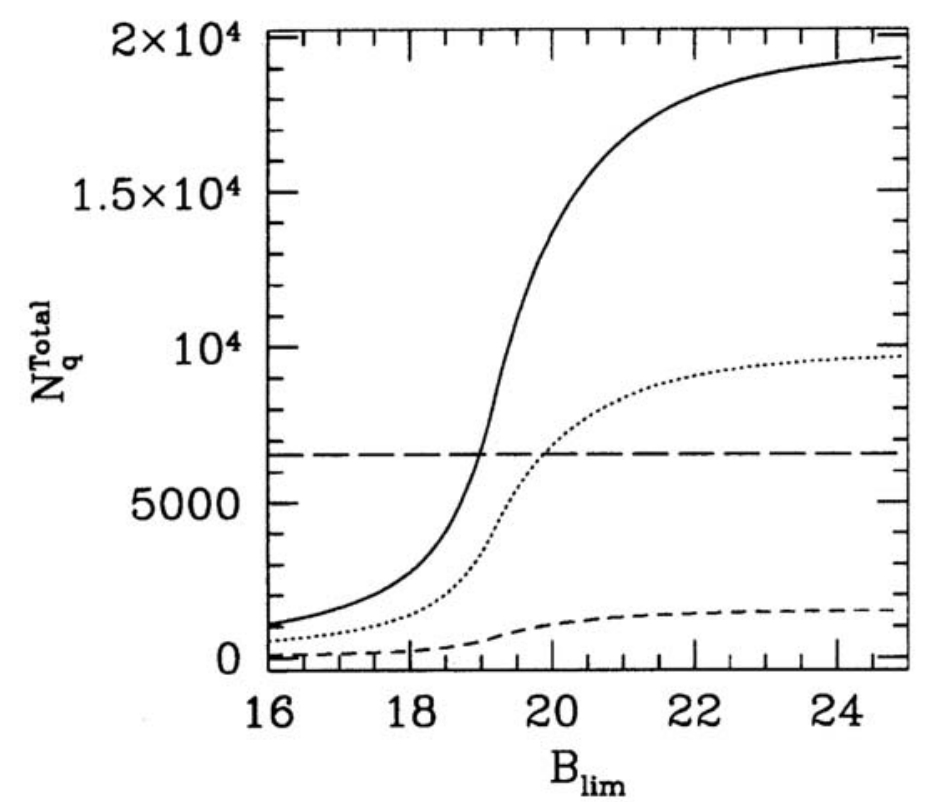

Figure 2. Total number of quasars $N_{q}^{\text {Total }}$ versus the limiting magnitude $B_{\text {lim }}$, expected in the surveyed sky area $S$ over which 50 new cases of GL ought to be identified. Unless stated otherwise, the number counts of quasars are taken from Hartwick and Schade (1990). The different curves refer to $\Omega_{0}=1, \lambda_{0}=0$ (full), $\Omega_{0}=0, \lambda_{0}=0$ (dotted), $\Omega_{0}=0$, $\lambda_{0}=1$ (dashed) and, finally, $\Omega_{0}=1, \lambda_{0}=0$ (long-dashed) with the number counts of quasars from Hawkins and Véron (1995).

\section{ILMT SPECIFICATIONS, THE CORRECTOR AND THE CCD}

At this time, we keep foreseeing two options for the CCD mosaics : either simultaneously observing in two wide spectral bands (cf. i'(760 nm) and r') with two $2 \mathrm{~K} \times 4 \mathrm{~K}$ CCDs, or four $2 \mathrm{~K} \times 2 \mathrm{~K}$ CCDs, or in a single wide spectral band (cf. i') using a single $4 \mathrm{~K} \times 4 \mathrm{~K}$ CCD. Considering first the case of the four $2 \mathrm{~K} \times 2 \mathrm{~K}$ CCDs, at the TDI rate, about 41 vectors are read per second for each of the four CCD chips. Including the over scan (see below), the vector length is about 2100 pixels. With a traditional coding on 2 Bytes for each of the 4 CCDs, this amounts to $41 \times 2100 \times 4 \times 2=0.69 \mathrm{MB} / \mathrm{sec}$ for the transmission rate, and a volume of raw data of about $20 \mathrm{~GB}$ per night on the acquisition machine. If instead, two $2 \mathrm{~K} \times 4 \mathrm{~K}$ CCDs or a single $4 \mathrm{~K} \times 4 \mathrm{~K}$ CCD chip is chosen, the volume of data will be reduced to $10 \mathrm{~GB}$ per night. The spatial resolution combined with the apparent motion of the sky translates into a time resolution of 0.025 sec per pixel. Therefore, an accuracy on UT of 0.01 or $0.001 \mathrm{sec}$ seems quite sufficient.

We provide hereafter the technical requirements for the procurement and manufacturing of a sub-system unit comprising the optical corrector and the detector to be integrated in the ILMT. We assume that this telescope will be installed on a site with median seeing of 1 arcsec, at a latitude of about $\pm 29^{\circ} 30^{\prime}$ (ESO's La Silla site in Chile or Devasthal site in India), and an altitude of about 2500 meters. Calls for tenders have recently been issued for the construction of both the optical corrector and the CCD camera.

\subsection{General specifications}

Figure 3 provides a sketch of the entire telescope system. Comparing the Liquid Mirror Telescope (LMT) to a conventional telescope, we see that they are similar with the outstanding exception that the LMT is always vertical; hence the mount is far simpler as the equipment feels a constant gravitational acceleration in the same direction. The top parts are similar to those of a conventional telescope, although simpler, consisting of an upper end holding the focusing system, an optical corrector and a CCD detector. All mechanical structures 
ought to be compliant with an operating temperature between $-20^{\circ} \mathrm{C}$ and $+20^{\circ} \mathrm{C}$. Tracking will be performed with the TDI technique.

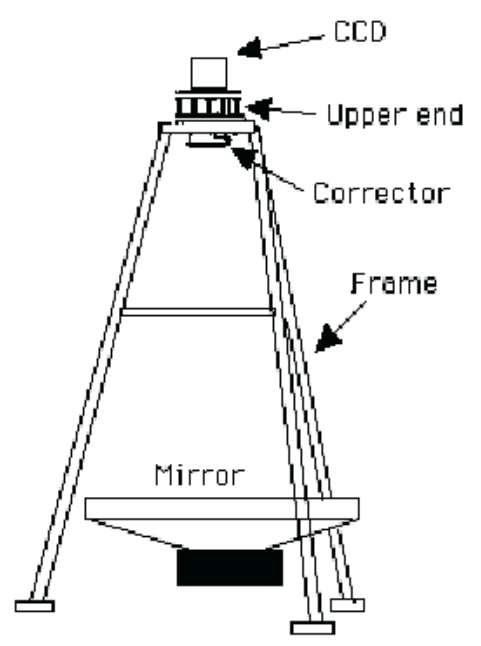

Figure 3: Telescope sketch

The system specifications are:

- Mirror diameter: 4 meters - f/2;

- Resolution: 0.6 arcsec FWHM;

- Corrector: FWHM = 0.2 arcsec (at focus);

- FOV: $30^{\prime} \times 30^{\prime}$ field;

- CCD: Thinned high QE, $4096 \times 4096$ equivalent mosaic (15 micron pixels);

- Filters: possibly i', r', g' in a split field configuration - i' permanently mounted;

- Location: $\pm 29^{\circ} 30^{\prime}$ latitude (Chile, La Silla or India, Devasthal);

- Life expectancy: 5 years.

\subsection{Error budget for the PSF}

All values refer to full-width at half maximum (FWHM). A median seeing of the order of 1 arcsec is assumed. The error budget for the PSF is accounted as follows :

- CCD pixels: $0.4 "$;

- Corrector: 0.2";

- Upper-end: 0.1";

- Mirror: $0.2 "$;

- Optical alignment errors: $0.1 "$. 


\subsection{Sub-system requirements : Mechanisms of the upper end}

The upper-end of the telescope holds the CCD detector and the optical corrector. Its purpose is to move the CCD detector up and down (z motion) for focusing purposes, to move the corrector and CCD sideways ( $\mathrm{x}-\mathrm{y}$ directions) and to move in tilt the optical axes of the CCD and of the corrector with respect to the optical axis of the primary mirror for optical alignment purposes. Its main components are listed below.

- Azimuth adjustment of the corrector: a mount will enable to rotate the corrector/detector around the optical axis. Its purpose is to align the TDI correction with the TDI distortion at the site location.

- Azimuth adjustment of the CCD: a mount will be used to rotate the CCD detector around the optical axis. Its purpose is to align the columns of the CCD in the East-West direction. The precision needed is 0.003 degree.

- Focusing z-mount: a z-mount is used to focus the detector. It moves the detector up and down (z-motion) with a precision of at least one micrometer. It may be possible to design the mechanical focusing system so that only the CCD detector is moved in z. The z-mount will be motor operated from the data room. It will have encoders and the associated electronics so that it can also be monitored from the data room. Provision will be made for a remotely controlled operation upgrade. It will include an automatic focusing system.

- X-Y mount (for the corrector): an $\mathrm{x}-\mathrm{y}$ mount must move the corrector sideways (holding it parallel to the optical axis of the mirror while doing so). The precision needed is 10 microns. It can be hand-operated but motor motions controlled from the data room are desirable.

- Tilt adjustment of the corrector: a three-point mount will hold the corrector to allow an adjustment in tilt of its optical axis with respect to the optical axis of the mirror. The angular precision needed is 0.001 degree. It can be hand operated but motor motions controlled from the data room are preferred.

- X-Y mount of the CCD: an X-y mount will position the center of the CCD on the optical axis of the mirror. The precision is at least $0.5 \mathrm{~mm}$. It can be hand operated.

- Tilt adjustment of the CCD: a three-point mount will hold the CCD to allow an adjustment in tilt of its optical axis with respect to the optical axis of the mirror. The angular precision needed is 0.001 degree. It can be hand operated. Temporal and temperature stabilities of the adjustments: all adjustments will be stable to the last quoted decimal over 6 months while the mechanical systems are exposed to temperature changes of $\pm 20^{\circ}$.

\subsection{Specifications of the Optical corrector}

The chosen corrector consists in an on-axis corrector capable of correcting the field of the CCD mosaic. The final images (for ideal point sources) will have a FWHM $<0.2 \operatorname{arcsec}(80 \% \mathrm{EE})$, including TDI distortion. Distortion shall be corrected so that the image center will be within $0.1 \operatorname{arcsec}$ (RMS) from the ideal position.

- FOV: $30^{\prime} \times 30^{\prime}$ square.

- Average $80 \%$ EE in the FOV: better than 0.2 arcsec in operational environment;

- Wavelength range: 450 to $950 \mathrm{~nm}$ (depending on the filters).

- Temperature range $1:-20^{\circ} \mathrm{C}$ to $+20^{\circ} \mathrm{C}$ (operational temperature).

- Temperature range $2:-25^{\circ} \mathrm{C}$ to $+30^{\circ} \mathrm{C}$ (temperature range that will not cause mechanical damage).

- Unique focus: the telescope focus has a unique value while observing anywhere from 450 to $950 \mathrm{~nm}$ in two separate filters.

- The effective focal length of the telescope will be lower than $10 \mathrm{~m}$. 


\subsection{Optical design}

The corrector system has been designed with computer optical design software. The design yields a system of 5 lenses that respect the specifications above. Tolerancing of the design has been performed. Attention has been paid to unique focus considerations (see item below). Ghosts and spurious reflections have also been minimized.

\subsection{Global transmission}

The global transmission of the corrector - without the filters - will be broadband with $\mathrm{T}=90 \%$ over the useful 450 - 950 nm range.

\subsection{Unique focus}

The telescope may simultaneously observe with two different (or similar) filters in a split field configuration. The telescope has thus a single focus setting, which may not be ideal for both bandpasses. Compensation will be made for the fact that this single focus may not be optimal for the three bands (e.g. g', r', and i'). The optical specifications apply for the wavelength range of any of the broadband filters g', r' or i'. Slight variations of focus can be tolerated between the different filters since they could be compensated by adding a glass slide above one of the filters.

\subsection{Vignetting}

The optics and mechanical mounts will not vignet the field specified above.

\subsection{Corrector cell}

The lenses are contained in a metallic structure. The structure will hold the optical elements in the positions specified by the design software with a precision determined by the tolerancing analysis. The cell will operate safely within the specified temperature range.

\subsection{Filters}

The filters will be standard g'r'i' filters. An i' filter will always be present. The science requirements make it highly desirable to observe simultaneously in two filters. Because variability in both positions and fluxes are important, one of the filters will be used in permanence while the others might be changed periodically. Allowance will be made for a filter holder with a split-field arrangement by which half of the CCDs on the mosaic observes with one filter and the other half observes with the second filter. The filters will be mounted as close as possible to the surface of the CCDs. Allowance will be made for mounting thick interference filters.

\subsection{CCD detector}

The CCD Quantum Efficiency will be as high as possible over a spectral range as wide as possible. It will therefore be desirable to use thinned backside illuminated CCDs. An equivalent $4 \mathrm{~K} \mathrm{x} 4 \mathrm{~K}$ CCD having 15 micron pixels that subtend 0.4 arcsec approximately covers a $30^{\prime} \times 30^{\prime}$ field. If not a single $4 \mathrm{~K} \times 4 \mathrm{~K} \mathrm{CCD}$, the CCD mosaic will have a gap in the middle of the field (in the read-out direction) as small as possible but large enough to permit the split-filter configuration. For instance, if we decide to use two EEV $2 \mathrm{~K}$ x $4.5 \mathrm{~K}$ CCD 42-90, their characteristics will be compliant with our specifications. 


\section{CONCLUSIONS}

First light of the $4 \mathrm{~m}$ International Liquid Mirror Telescope is foreseen before the end of 2007. Possible site locations are either in the Atacama desert (latitude near $-29^{\circ} 30^{\prime}$ ) or in North-East India (Nainital Hills, latitude near $\left.+29^{\circ} 30^{\prime}\right)$. The ILMT will monitor, for about five years, the same trip of sky, night after night, detecting all photometrically and astrometrically variable faint objects (typically i' $\sim 22.5$ ). The scientific applications justifying the construction of such a telescope, an instrument description and a brief description of this multinational undertaking have been presented. For the case of multiply imaged quasars, we find that direct imagery with the 4m ILMT should lead to the detection of at least 50 new gravitational lens systems. The natural possibility to photometrically monitor these at daily intervals offers a unique opportunity to define a sub-sample of interesting lenses with reliable geometrical parameters, time delay measurements and/or microlensing signatures for further astrophysical and cosmological applications. The possible construction of an array of several LMTs, working in phase at a single wavelength or independently at different wavelengths, is also foreseen in the near future.

\section{ACKNOWLEDGMENTS}

The ILMT project is supported by contract IUAP P5/36 "Pôle d'Attraction Interuniversitaire", by the "Communauté Française" and the "Région Wallonne de Belgique", by the "Fonds National de la Recherche Scientifique" and the "Liège University", Belgium.

\section{REFERENCES}

[1982] Borra E. F. : 1982, JRASC 76, 245

[1992] Borra E. F., Content R., Girard L., Szapiel S., Tremblay L. M., Boily E.: 1992, Ap. J. 393, 829

[1992] Borra E. F., Content R., Girard L. : 1992, Ap. J. 393, 829

[1995] Borra E. F., Content R., Girard L.: Robotic Observatories. Edited by Michael F. Bode. Wiley-Praxis

Series in Astronomy and Astrophysics, Chichester, New York: Wiley, 1995, p.125

[1997] Borra E. F., Ferrari M., Girard L., Moretto G., Tremblay G., Lemaitre G. R.: 1997, SPIE.2871..326B

[1998] Cabanac R., Borra E.F., Beauchemin M.: 1998, Ap. J. 509, 309

[1979] Chang K., Refsdal S.: 1979, Nature 282, 561

[2002] Claeskens J.-F., Surdej J.: 2002, Astron. Astrophys. Review 10,263-311

[1990] Hartwick F. D. A., Schade D.: 1990, ARA \& A 28, 437

[1995] Hawkins M., Véron P.: 1995, Monthly Notices Roy. Astron. Soc. 275, 1102

[1994] Hickson P., Borra E. F., Cabanac R., Content R., Gibson B. K., Walker G. A. H.: 1994, Ap. J. 436, L201

[1993] Hickson P., Gibson B. K., Hogg D. W. : 1993, PASP 105, 501

[1997] Hickson P.: 1997, JRASC, 91, 58

[1998] Hickson, P., Mulrooney, M.K.: 1998, Ap. J. Suppl., 115, 35 preprint

[1992] Kayser R.: 1992, Lecture Notes in Physics 406, 143

[2004] Kochanek C.S., Schneider P., Wambsganss J.: 2004, Gravitational Lensing: Strong, Weak \& Micro. Proceedings of the 33rd Saas-Fee Advanced Course, G. Meylan, P. Jetzer \& P. North, eds (Springer-Verlag: Berlin)

[1991] Lilly S. J., Cowie L. L., Gardner J. P.: 1991 Ap. J. 369, 79

[] Mailly E.: 1872, De l'Astronomie dans l'Acadmie Royale de Belgique, Rapport Séculaire, 1772-1872, 99

[1991] Metcalfe N., Shanks T., Fong, R., Jones L. R.: 1991, MNRAS. 249, 498

[1996] Ninane N.M., Jamar C.A. : 1996, Applied Optics 35, No.31, 6131

[1964] Refsdal S.: 1964, Monthly Notices Roy. Astron. Soc. 128, 307

[1993] Surdej J., Claeskens J.-F., Crampton D., Filippenko A.V., Hutsemékers, D. et al.: 1993, Astron. J. 105, 2064 